\title{
Soft Microscopy of Negative Stained Soft Materials: Balancing Dose Rate and Sample Damage
}

Chamille Lescott ${ }^{1}$, Roberto dos Reis ${ }^{2}$, Mallika Modak ${ }^{3}$, Evan $\operatorname{Scott}^{3}$ and Vinayak Dravid ${ }^{2}$

${ }^{1}$ Department of Materials Science \& Engineering, Northwestern University, United States, ${ }^{2}$ Department of Materials Science and Engineering, Northwestern University, United States, ${ }^{3}$ Department of Biomedical Engineering, Northwestern University, United States

Electron energy loss spectroscopy (EELS) and energy-dispersive x-ray spectroscopy (EDX) in a scanning electron microscope (STEM) are promising methods for the elemental and electronic structural analysis of soft biological materials [1]. When preparing these soft materials for STEM, the sample preparation and imaging method impacts the information one can gain from the EELS/EDX spectrum. Here we ponder the advantages and disadvantages of using a stained sample for EELS/EDX analysis. Negative staining can increase the background in an EELS/EDX spectrum making an unstained sample preferable. However, staining provides morphological information that can give a clearer picture of the structure when paired with an EELS/EDX map. By imaging stained and unstained samples at room and cryogenic temperatures, we aim to tune the critical dose, impacting the quality of the EELS/EDX spectrum. This work analyzes the role that sample fixation may play in preparing soft biological samples for analytical microscopy techniques.

The sample used in this work is a poly(ethylene glycol)17- $b$-poly(propylene sulfide) 17 (PEG- $b$-PPS) bicontinuous nanosphere $(\mathrm{BCN})$, which is a self-assembled nanostructure and promising drug delivery platform [2]. The BCN demonstrates cubic ordering due to the system of non-intersecting amphiphilic bilayers of PEG- $b$-PPS polymer within the nanostructure (Figure 1a) [3][4]. The sample is beneficial for our analysis because evidence of this internal structure is present in a negative stained sample but not in a dried unstained sample. Thus, at the outset staining provides distinct structural information that we can compare to the results of EELS/EDX maps. The BCN also contains sulfur from the propylene block of the PEG- $b$-PPS diblock that may be detected by EDX and EELS. Unstained samples were prepared by drop-casting and drying the BCNs on hydrophilized copper formvar/carbon TEM grids. Negative stained samples were prepared with $1 \%$ uranyl formate on hydrophilized grids. Initially, the samples were imaged on a Hitachi-2300 dedicated STEM operated at 200kV. In the unstained sample, the sulfur (K alpha, 2.309 $\mathrm{keV}$ ) EDS peak is detected within the BCN and is likely responsible for the BCN's contrast in the $\mathrm{z}-$ contrast weighted annular darkfield image (ADF) (Figure 1b-c). In the negative stained sample, a bubblelike morphology is present with high-Z stain pooling inside the BCN. For this sample, the interaction with the stain provides information about the BCN's structure that, when correlated with the sulfur mapping, can elucidate details of the morphology (Figure 1d-e). Notably, the negative stain increases the background in the EDX spectrum and calculated sulfur maps (Figure 1f-g). EDS maps were then processed by binning with a 11x11 kernel size, which resulted in maps that improved the sulfur signal relative to the background but decreased spatial resolution. Here, we see an example of the challenges facing the use of a negative stain with spectroscopy. However, if we achieve higher resolution sulfur maps of these negative stained samples that we can compare to the unstained sample, we will gain an understanding of how the BCN's structure of internal channels may be interacting with the stain to produce the distinct morphology. 
Intending to generate higher resolution elemental maps, sample EELS spectra were collected on a JEOL ARM200CF Aberration-Corrected TEM operated in STEM mode at 200kV and equipped with Gatan K2/GIF quantum system that allows us control of electron dose via fast acquisition EELS spectra up to 400fps. Samples were analyzed at cryogenic and room temperatures with the expectation that lower temperatures will allow for the use of higher dose rates without excessive damage to the sample. Preliminary analysis of the plasmon peak and calculation of $t / \lambda$, which provides a measurement of the relative thicknesses within the image, confirms that the unstained $\mathrm{BCN}$ is thicker than the grid background (Figure 2a-c). $t / \lambda$ analysis of the negative stained BCN shows that the sample is embedded in a layer of stain that is of uniform thickness across the grid, which again highlights the distinct difference in sample preparation techniques (Figure 2d-f). Signals from both the stain and the sample contribute to the EELS spectrum and require careful analysis and future deconvolution. Preliminary results confirm that with EELS both sulfur and carbon can be detected in the unstained sample (Figure $2 \mathrm{~g}$ ).

Soft microscopy is an expanding field focused on merging analytical techniques such as EELS and EDS with appropriate sample preparations for the targeted analysis of soft structures. Developing a better understanding of how sample preparation, including negative staining techniques, can work with spectroscopy to improve our knowledge of soft systems involves the careful tuning of the electron dose, contamination, sample damage, and the signal-to-noise ratio of the collected spectra. We will present data on how cryogenic imaging allows for the use of higher doses with minimal sample damage and the collection of higher quality EELS/EDS data. Additionally, the work may be expanded to further investigate the carbon edge. When damaging the soft material, the electron beam can generate carbon $\Pi-$ bonds, which changes the shape of the carbon edge [5]. Since no ח-bonds are present in the sample initially, changes to the carbon edge can indicate sample deterioration under different imaging and sample preparation conditions.

The techniques and analysis highlighted here are transferable to the study of other soft and biological materials, especially for non-crystalline structures where damaging processes cannot be accessed via electron diffraction. We aim to investigate how we can use multiple analytical techniques and sample preparation methods to gain a more in-depth understanding of soft structures and expand the information one can gain from a fixed sample [6].

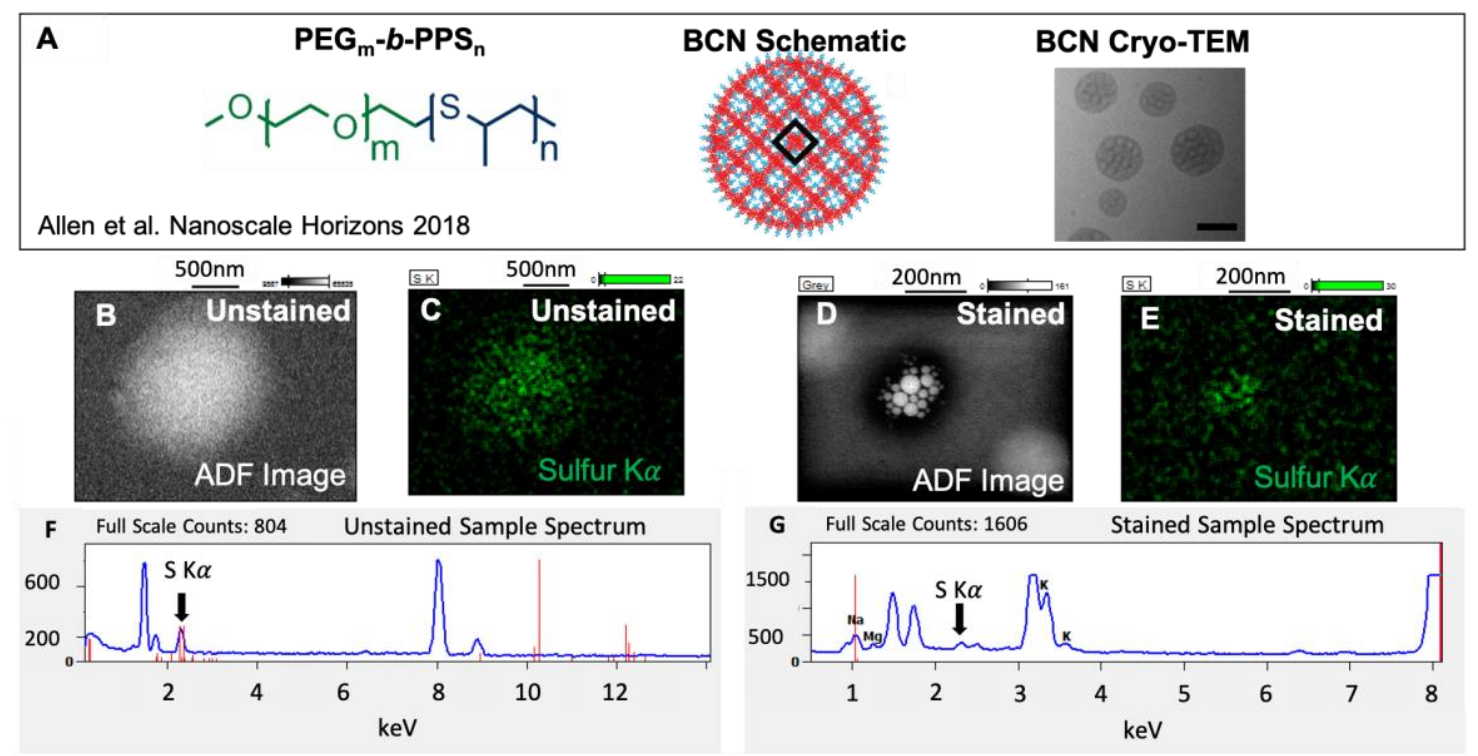


Figure 1. a) Published literature on BCNs show the complexity of the structure and evidence of aqueous channels in cryo-TEM images [4] b) ADF image of unstained BCN. (c) Binned sulfur map showing the concentration of the sulfur signal inside the unstained BCN. d) ADF image of stained BCN. (e) Binned sulfur map showing the concentration of sulfur signal inside the stained BCN. (f) EDS spectrum from the unstained sample map confirms the presence of a detectable sulfur peak. (g) Notably, the EDS spectrum for the stained BCN contains more background peaks from the stain which contributes to the background in the EDS map.
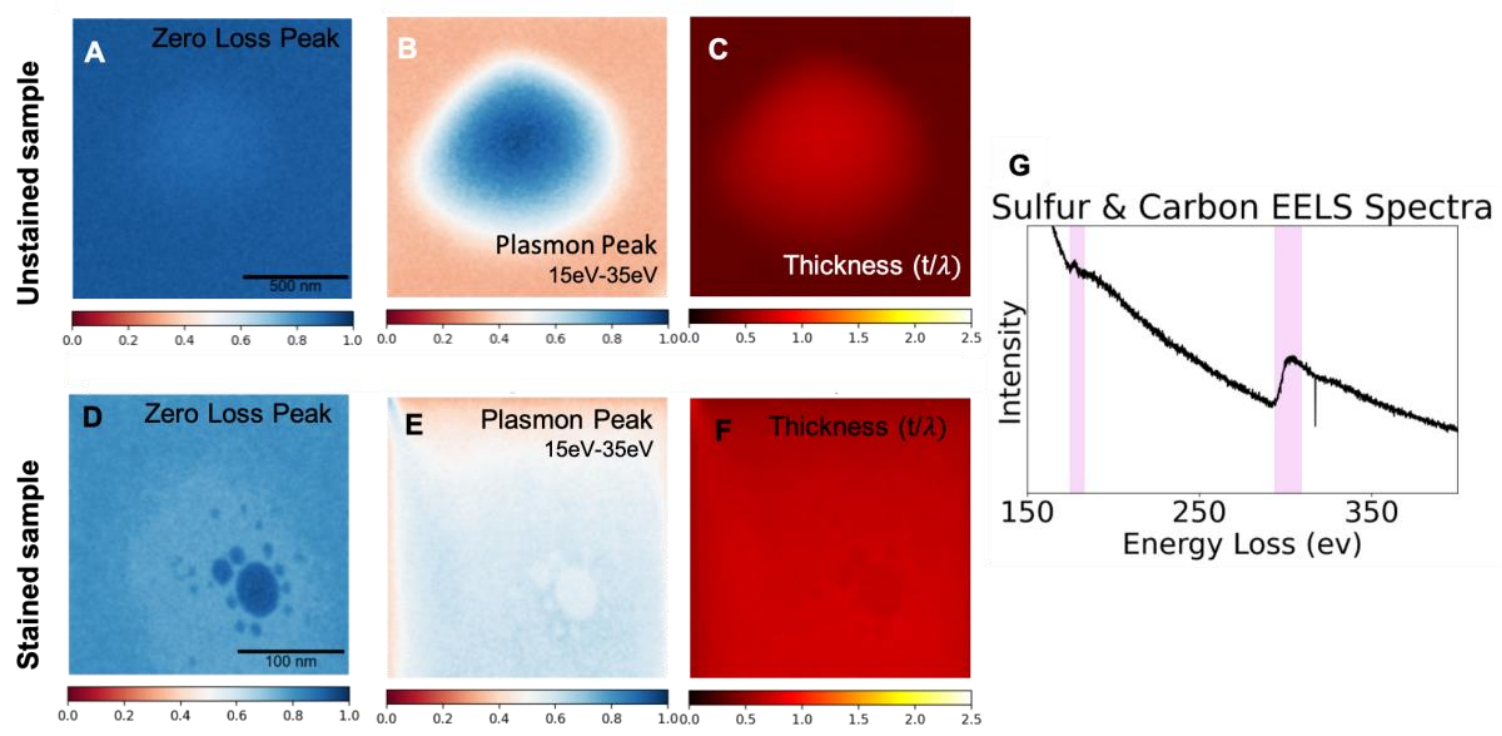

Figure 2. (a/d) Brightfield images generated from integration of the zero loss peak for the unstained (a) and stained (d) sample. (b) Corresponding plasmon map of an unstained sample showing a clear difference between the background and the BCN. (c) $t / \lambda$ thickness map displaying the relative thickness of the features. (e) Negative stained plasmon map displaying little distinction between the background and the sample. (f) Evidence that the stained sample is embedded in a layer of fairly uniform thickness is seen in the thickness map. (g) Confirmation that a sulfur and carbon signal is detectable with EELS in the unstained sample is shown in the plot of a spectrum averaged across the image.

\section{References}

[1] Sousa, A. A., \& Leapman, R. D., Ultramicroscopy, 123, (2012), 38-49.

[2] S Bobbala et al, Nanoscale, 12 (2020), 5332-5340

[3] S Bobbala, SD Allen, and EA Scott, Nanoscale 10 (2018), p. 5078-5088.

[4] Allen, Sean D., et al. Nanoscale horizons 4 (2019): 258-272.

[5] Yakovlev, S., \& Libera, M. Micron, 39(6) (2008)., 734-740.

[6] This work was primarily supported by Air Force Office of Scientific Research (AFSOR) FA9550-171-0348 (subproject for Reconfigurable Matter from Programmable Atom Equivalents) and CABN(AFRL) FA8650-15-2-5518 (Statistics and Dynamics of Soft Biological Structures and Hybrid SoftHard Assemblies). Additional funding from ARO/MURI W911NF1810200 (Dravid Subproject for Stimuli-Responsive Control of Protein-Based Molecular Biology). The research of Evan A Scott is supported by the National Science Foundation (CAREER-1453576), the National Institutes of Health Director's New Innovator Award (NHLBI 1DP2HL132390-01), and the National Institute of Allergy and Infectious Disease (NIAID 5R01AI145345-02). This work made use of the BioCryo facility of 
Northwestern University's NUANCE Center, which has received support from the SHyNE Resource (NSF ECCS-2025633), the IIN, and Northwestern's MRSEC program (NSF DMR-1720139). 